The Open Materials Science Journal
CrossMark
Content list available at: www.benthamopen.com/TOMSJ/
DOI: $10.2174 / 1874088 \mathrm{X} 01812010014$

RESEARCH ARTICLE

\title{
Novel Approach to The Design of Sound Insulating Composites by Means of a Non-linearly Extrapolated Master Curve
}

\author{
Cecchini Federico, Cherubini Valeria, Francesco Fabbrocino ${ }^{*}$ and Francesca Nanni \\ Pegaso University, piazza trento e trieste - Napoli, Italy
}

Received: April 19, 2018

Revised: May 23, 2018

Accepted: June 02, 2018

\begin{abstract}
:
Background:

The increasing use of composite structures with a high stiffness-to-weight ratio in commercial vehicles has brought about a reduction in fuel consumption but, on the other hand, has significantly increased noise transmission particularly in case of thin and lightweight structures. Noise is a primary issue for commercial vehicles, such as airplanes, helicopters and cars. The present research deals with the use of smart materials, as Shear-Thickening Fluids (STF, or dilatants) in view of manufacturing elements with increased sound insulation properties.
\end{abstract}

Methods:

The response of a sandwich material with the STF core was investigated both experimentally and numerically, by choosing the Sound Transmission Loss (STL) of the composite structure as the figure of merit.

The experimental investigation was focused on the manufacturing of a sandwich structure made of metallic skins and a STF core that was successively characterized by sound insertion loss measurement.

The numerical investigation was carried out by using a Generalized Transfer Matrix Method (GTMM) and a Statistical Energy Analysis (SEA) in view of selecting the fluid capable of granting the highest acoustic transmission loss.

Results:

Finally, the test results were compared to the numerical results, showing a noticeable agreement. The used STF showed increasing viscosity at increasing shear rates.

Keywords: Vibroacoustics, SEA, Rheology, Non newtonian fluids, Nanocomposites, Master curve.

\section{INTRODUCTION}

The use of shear thickening fluids (STFs or dilatants) in Stab-resistant and impact absorbing applications has been thoroughly studied in recent years [1]. These Non-Newtonian fluids present an abrupt increase in the apparent viscosity with the imposed shear rate and can therefore be successfully used for the manufacturing of passive smart materials, advanced composite systems capable of altering their constitutive law basing on the applied stress/strain field.

From a microscopic perspective, this transition is caused by the flocculation of the particles that, when the imposed shear rate reaches a critical value, flocculate and form complex aggregates known as hydroclusters. By altering the composition of these fluids in terms of carrier fluid, filler and filler concentration, from a macroscopic point of view, this structural modification induced as a byproduct of a certain strain field and a certain regular frequency spectrum can be used to calibrate the vibration dampening properties of these materials and, as consequence, their sound insulation

* Address for correspondence to this auther at the Pegaso University, piazza trento e trieste, Napoli, Italy, Tel: +081191383; E-mail: FRANCESCO.FABBROCINO@UNIPEGASO.IT 
properties.

From a Vibroacoustic perspective, particularly for coupons with increased sound insulation capabilities, the peculiar vibration dampening properties of STFs can be best employed by recurring to sandwich structures in constrained layer damping configuration (CLD), where the STF is interposed as the core of the sandwich structure and the outer skins are made of a much stiffer material, such as Aluminum.

In fact, there is a nontrivial difference from the extensional layer damping configuration, in which the polymer is used as the outer skin and undergoes extensional strains only.

On the other hand, in this structural configuration, the outer skin impacted by sound pressure energy induces really high shear strains in the core of the sandwich, as the value of its storage modulus is orders of magnitude lower than that of the top/bottom layers [2].

The shear strains are converted into heat by the core undergoing the aforementioned microstructural transformations, reducing therefore the sound radiating from the opposite skin.

The feasibility of the use of STFs for low regular frequency vibration damping of sandwich systems was already proved in previous researches [3, 4]. However, to the best of our knowledge, there is a lack of sound insulation characterization for STFs and, in general, for sandwich systems comprising Non-Newtonian cores. One of the main issues related to the choice of these materials derives from the lack of tools capable of measuring the rheological properties of Non-Newtonian fluids at acoustically relevant frequencies (i.e. 1-20 kHz).

In fact, the most commonly available rheometers and DMTA systems allow users to perform measurements at frequencies, generally speaking, lower than $400 \mathrm{~Hz}$ and, in particular, for rheometers, this value is lower, attesting in most cases at $20 \mathrm{~Hz}$. On the other hand, commonly used time-temperature superposition techniques, such as the WLF, were initially brought forward for Creep compliance and Stress relaxation measurements and typically do not provide satisfactory data for acoustic phenomena [5].

Furthermore, most Non-Newtonian fluids are characterized by a Continuous relaxation spectrum with a wide band of relaxation times, that complicates the extrapolation of the high regular frequency response even by recurring to more numerically complex techniques [6]. This problem is even more concerning for STFs, that present an anomalous behavior, when compared to simpler Non-Newtonian fluids [7], as the effects of shear strains and regular frequency cannot be superimposed and they require more complex rheological characterization because of secondary rheological phenomena [8].

In light of the aforementioned critical points, during the first part of this work we choose to adopt a different approach for the modeling of the acoustic constitutive law of our samples. We used numerical methods derived from optics [9], in order to extrapolate the high regular frequency response of our samples basing on the available low regular frequency data only.

We carried out this analysis with an optimized [10 - 12] combination of fillers and carrier, and we varied the filler concentration.

During the second part of this research work we focused instead on the numerical modeling of the sound insulation properties of the fully assembled CLD sandwich.

In particular, we modeled the Sound Transmission Loss $(S T L)$ by using a Statistical approach that takes into account the modal contribution of the various natural modes manifested by the vibrating coupon.

For the constitutive law used in the model, we recurred to the previously extrapolated high regular frequency rheological data as a tool to obtain the discrete relaxation spectrum of the STF and a Maxwell-Boltzmann constitutive law, where each relaxation time and peak corresponds to a certain combination of spring-dashpot present in a certain branch of the model.

Finally, we compared the results obtained by varying the filler concentration with a well-known in acoustics and less refined model (Generalized Transfer Matrix Method - GTMM) and we validated our model by comparing the results for both models with sound insertion loss data. 


\section{MATERIALS AND METHODS}

\subsection{Rheological Analysis}

During the first part of the experimental campaign, we focused on the manufacturing of the rheological samples and on the definition of an experimental protocol to produce rheologically coherent data, necessary for the following calibration of the acoustic constitutive behavior.

The selected STF was a Polyethylene glycol (Sigma Aldrich PEG200) with fumed silica (Sigma Aldrich $12 \mathrm{~nm}$ ) at different amounts: 20-25-30-35-40 weight-to-weight ratios (wt) according to [13]. In the following, this value will be always implicitely expressed as a percentage. Silica nanopowder was initially dispersed in ethanol, then PEG was added and the resulting dispersion was heated at $80{ }^{\circ} \mathrm{C}$ and magnetically stirred up to solvent complete evaporation.

The prepared nanocomposite STF were then characterized by means of a strain rate-controlled rheometer. (Malvern Kinexus LabPro+ rheometer). The fixture was a conical plate. All the samples (volume $=0.25 \mathrm{ml}$ ) were tested at $25^{\circ} \mathrm{C}$ with a $0.8 \mathrm{~mm}$ gap under the plate.

In previous works $[4,14]$ it was demonstrated that STFs do not obey to the rule of Delaware-Rutgers and, therefore, the low strain rheological analysis cannot be carried out by means of a single parameter combining both the oscillation angular frequency and the oscillation amplitude [15] (i.e. dynamic shear strain $\gamma=\gamma_{0} * \sin (\omega t)$ or a dynamic shear rate $\left.\gamma=\gamma_{0} * \omega\right)$.

During the first phase of the rheological experimental campaign, we focused on the strain sweep analysis at a constant angular frequency (to cite a few, $[16,17]$ ).

This first analysis allowed us to identify the shear rate associated with the linear viscoelastic behavior of the fluid (identifiable by the approximately linear region of the $G$ ' spectrum).

To this aim, all the samples were pre-conditioned for 1 minute at a constant strain rate of $1 \mathrm{~s}^{-1}$, in order to remove pre-existing stress fields. Subsequently a 2-step oscillatory rheological analysis was carried out.

First of all, the angular frequency was fixed at different values and a shear strain sweep was carried out, to evaluate the storage and loss moduli for all the samples. The testing angular frequency was $100 \mathrm{rad} / \mathrm{s}$ for all samples.

Finally, we carried out an angular frequency sweep analysis with a shear strain imposed. This parameter corresponds to a value slightly inferior to the upper bound of the preliminarily evaluated linear viscoelastic region. This value is highly dependent on the filler concentration and must be therefore derived from the first analysis.

\subsection{Acoustical Analysis}

For the evaluation of the sound insulation properties of the STFs, we used a sandwich in constrained layer damping. The outer skin of these coupons were made of aluminum and the core was made of the Non-Newtonian fluid.

More in detail, each STF sample was inserted between two 470mm x 340mm aluminum Al6060 skins Table 1, to form a $3 \mathrm{~mm}$ thick core (Fig. 1).

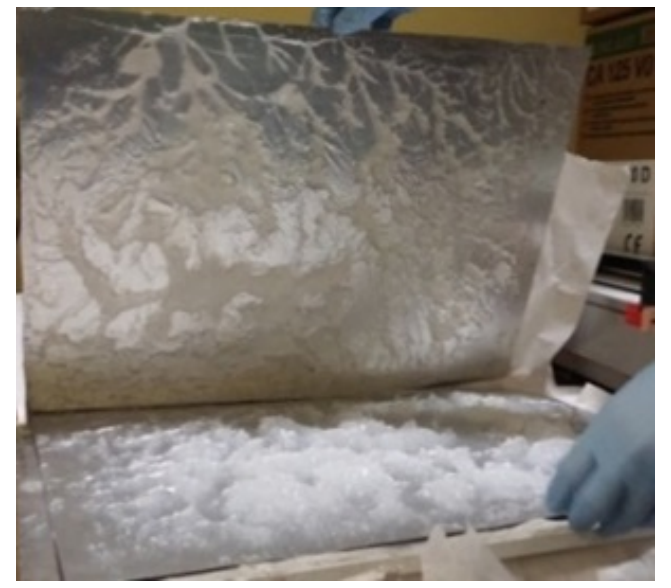

Fig. (1). Acoustical sample preparation: The stf core (white paste) is interposed between two aluminum skins. 
Table 1. Al6060 properties.

\begin{tabular}{|c|c|}
\hline Dimensions & $470 \mathrm{~mm} \times 340 \mathrm{~mm}$ \\
\hline thickness & $0.5 \mathrm{~mm}$ \\
\hline Density & $2700 \mathrm{~kg} / \mathrm{m}^{3}$ \\
\hline
\end{tabular}

The sandwich was then sealed with a thin layer of silicone sealant. Afterwards, all the samples were weighted, to assure consistency, and all the samples were found to have a surface density of $5.40 \pm 0.01 \mathrm{~kg} / \mathrm{m}^{2}$.

The sandwich used exhibits the following characteristics (Table 2):

Table 2. Sandwich panel properties.

\begin{tabular}{|c|c|}
\hline Dimensions & $470 \mathrm{~mm} \times 340 \mathrm{~mm}$ \\
\hline Total Thickness & $4 \mathrm{~mm}$ \\
\hline Surface Density of the Sandwich & $5.40 \mathrm{~kg} / \mathrm{m}^{2}$ \\
\hline
\end{tabular}

The experimental setup consisted of a $6.4 \mathrm{~m}^{3}$ reverberation chamber, suggesting the lowest testing regular frequency and an upper testing regular frequency of $20 \mathrm{kHz}$.

The sample was inserted in a clamp bolted to the front wall of the chamber, thus materializing the baffled conditions of the sample. The effective area of the sample was $420 \mathrm{~mm}$ x $297 \mathrm{~mm}$ (corresponding to the surface area of an ISO A3 sheet).

The audio acquisition equipment used can be summed up as follows:

- Two laboratory microphones Superlux ECM 888 B 1/4" with the following characteristics (Table 3):

\section{Table 3. Microphone characteristics.}

\begin{tabular}{|c|c|}
\hline Polar Curve & omnidirectional \\
\hline Frequency Response & $20-20000 \mathrm{~Hz}$ \\
\hline Signal to Noise Ratio & $72 \mathrm{~dB}$ \\
\hline Sensitivity (at 1000Hz) & $94 \mathrm{~dB} \mathrm{SPL}$ \\
\hline
\end{tabular}

- An 80-watt pre-amplified speaker (JBLPRX800) with a flat response (without a prevailing content between 50 $\mathrm{Hz}$ and $20000 \mathrm{~Hz}$ ).

- A dual mode acquisition card Midtech Audiolink III (two microphone input-ports and a STEREO or dual MONO audio output-ports).

The experimental setup consists of a 2-step sound pressure level measurement (Fig. 2):
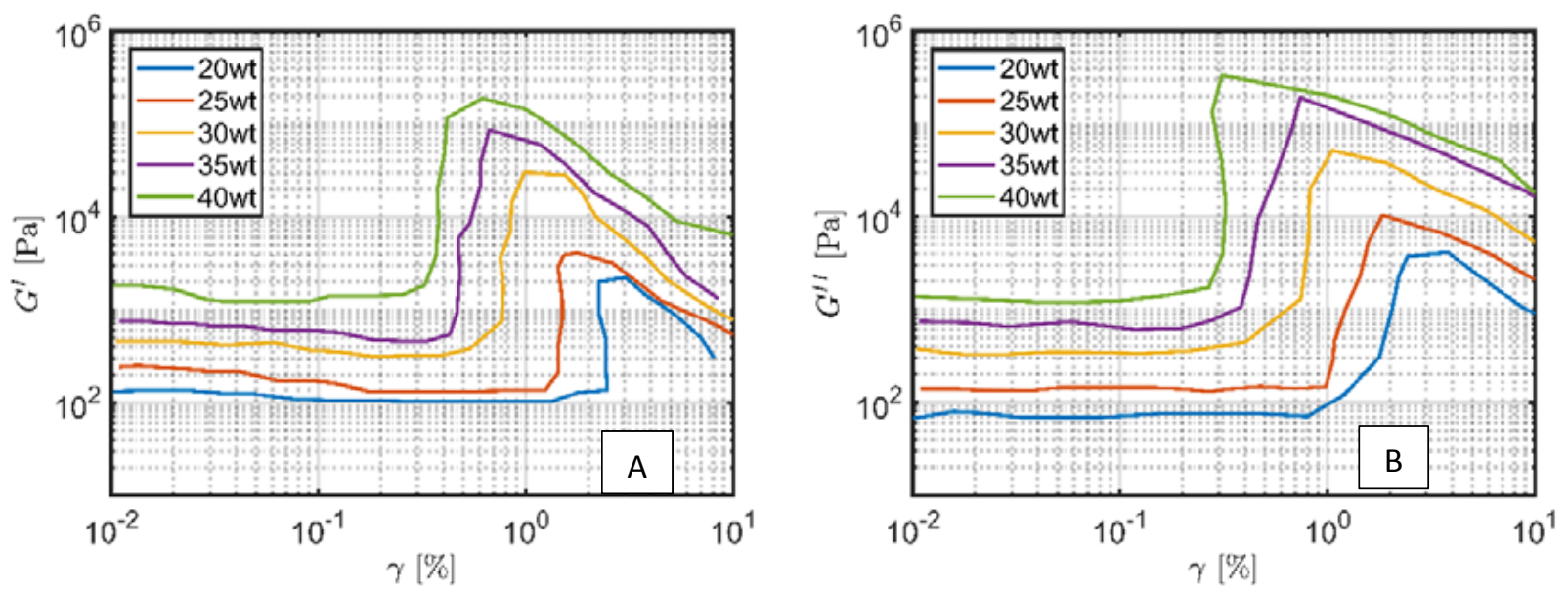

Fig. (2). Insertion loss measurement setup. 
1. During the first phase, we measured the sound pressure level outside the chamber with the holder in the "open" configuration (without the sample);

2. During the second phase, we measured the sound pressure level outside of the chamber with the samples inserted ("closed" configuration).

\section{RESULTS}

\subsection{Rheological Results}

As it can be clearly inferred in the reported graphs, Figs. (3 and 4 ) the viscous behavior of the suspension is more remarkable than the elastic one, as the loss modulus tends to overcome the storage modulus over the analyzed angular frequency range.
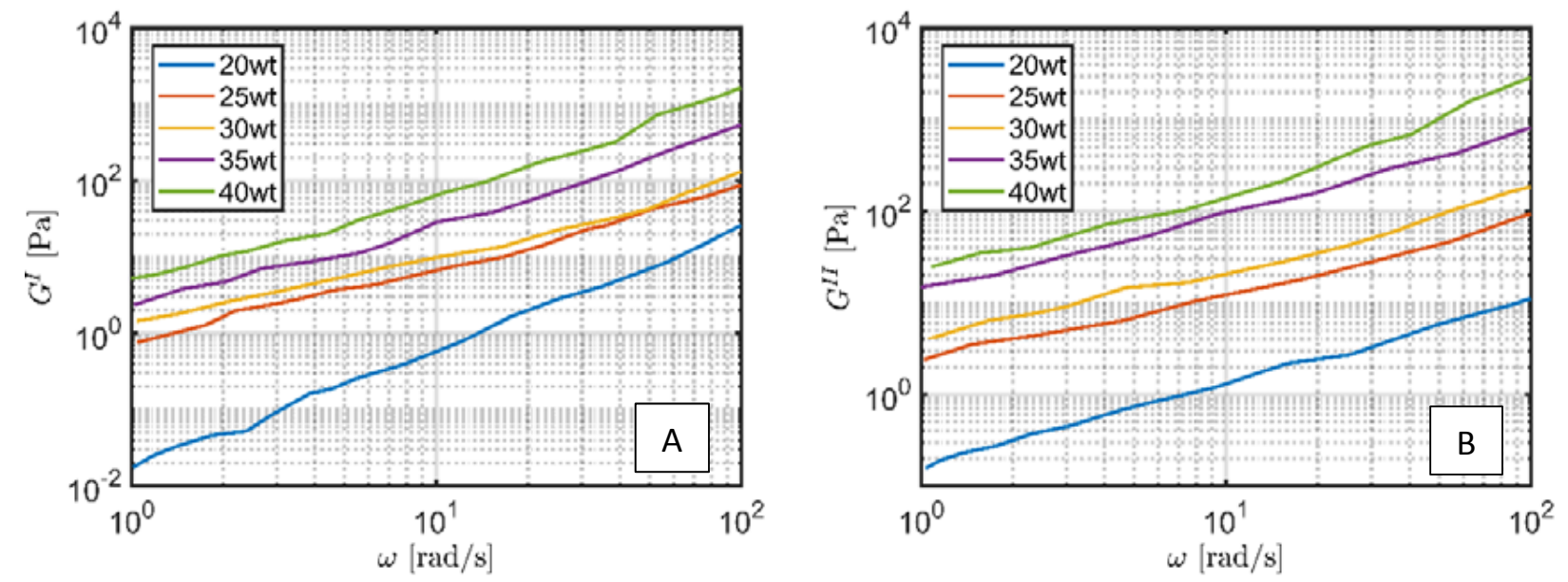

Fig. (3). Storage (A) and loss modulus (B) in strain sweep at $100 \mathrm{Rad} / \mathrm{S} v s \mathrm{Wt}$.

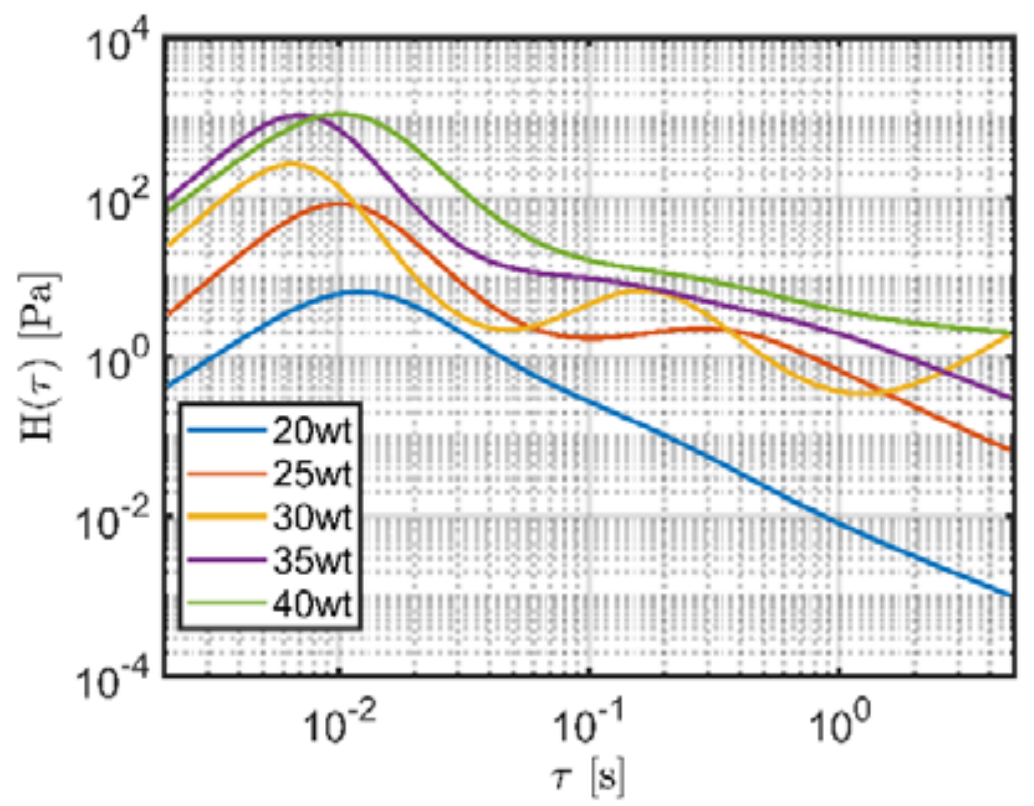

Fig. (4). Storage (a) and Loss modulus (b) in angular frequency sweep vs wt.

Secondarily, the linear viscoelastic region tends to shorten as we increase the filler concentration.

This final step provided us with the actual low angular frequency data was used for the numerical extrapolation of the discrete relaxation spectrum. 


\subsection{Extrapolation of the Rheological Properties at Higher Frequencies}

Commonly available laboratory rheometers, such as ours, allow users to evaluate the rheological properties of fluids at angular frequencies lower than $100 \mathrm{rad} / \mathrm{s}$.

Consequently, the dependence of the shear moduli of our fluids with angular frequency can be measured directly only up to this upper bound. On the other hand, acoustically relevant regular frequencies in most noise control applications span between $20 \mathrm{~Hz}$ and $20000 \mathrm{~Hz}$. Therefore, it was necessary to extrapolate the shear moduli by means of a Rheogram (i.e. a Master curve) at higher frequencies. Previous works show that the calculation of the discrete relaxation spectrum can be used to evaluate the shear moduli outside of the regular frequency range actually taken into account in the rheological tests.

In particular, we wanted the acoustic constitutive behavior of the STF by means of a complete yet computationally inexpensive law. We choose a Maxwell- Boltzmann generalized approach [18], where each branch of the model can be traced back to a certain main relaxation time of the discrete relaxation spectrum of the STF.

In order to extrapolate these values, it was necessary first of all to solve the nonlinear regression of the spectrum from the shear moduli. This is a hill-posed problem, known in mathematics as Tikhonoff inverse regression.

This problem revolves around the resolution of an integral equation, known as Fredholm' s integral equation, where the terms indicated by the subscript $\sigma$, are experimental values obtained by rheological measurements, therefore affected by an unknown error.

\subsection{Equation 1 - General Form of the Target Function}

Where:

- $\lambda$ denotes the regularization parameter of the target function;

- $G^{\prime}, G^{\prime \prime}$ are respectively the theoretical storage and loss moduli;

- $G_{\sigma i}^{\prime}, G_{\sigma i}^{\prime \prime}$ are respectively the experimental storage and loss moduli;

- $\sigma_{i}^{\prime}, \sigma_{i}^{\prime \prime}$ denote respectively the errors on the storage and loss moduli;

- $H$ denotes the continuous relaxation spectrum with relaxation times $\tau$.

The constraints linking the shear modulus to the loss modulus derive from Kramer's-Krönig integral equations:

\subsection{Equation 2- Kramer's-Krönig Integral Equations}

In approximate terms, more useful for the following numerical modeling:

\subsection{Equation 3- Kramer's-Krönig Integral Equations (Finite Summation)}

Where:

- $\omega$ denotes the angular frequency;

- $G^{\prime}, G^{\prime \prime}$ denote the relaxed storage and loss moduli;

- $h_{i}, \omega_{i}$ denote the associated terms of the discrete relaxation spectrum.

Both the spectral and differential terms under the integral were changed to $10^{\mathrm{H}(\omega)} \mathrm{d}(\operatorname{In}(\omega))$, in order to ease convergence [19].

The regularization problem was solved by using Weese' s algorithm [20, 21]. In order to obtain highly resolved spectra, the experimental data was preliminarily discretized by recurring to Kontogiorgos' method [22, 23], therefore by preliminarily creating a meshgrid of the kernel data in MATLAB.

\subsection{Validation of the Proposed Approach}

The numerical approach proposed in the previous paragraph was applied to the data extrapolated by the angular frequency sweep analysis Figs. (5 and $\mathbf{6})$. 


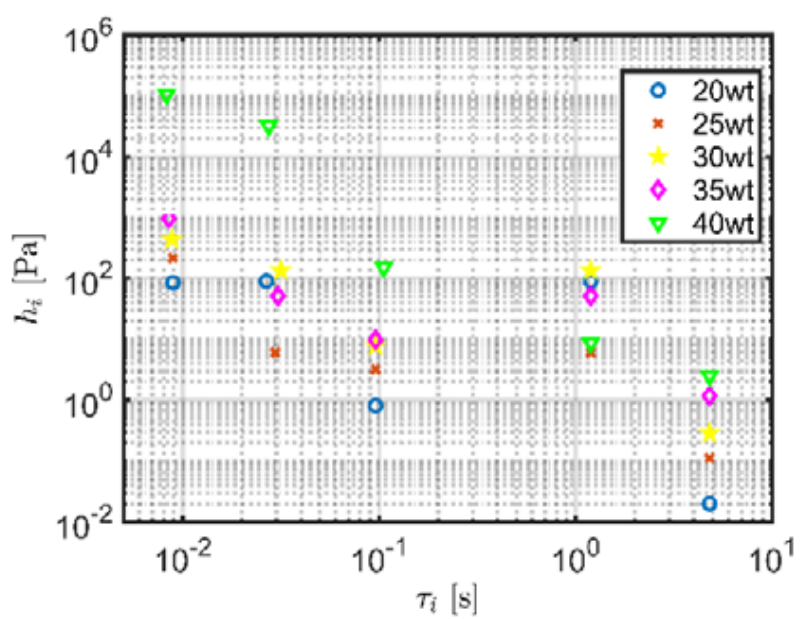

Fig. (5). Continuous relaxation spectrum.
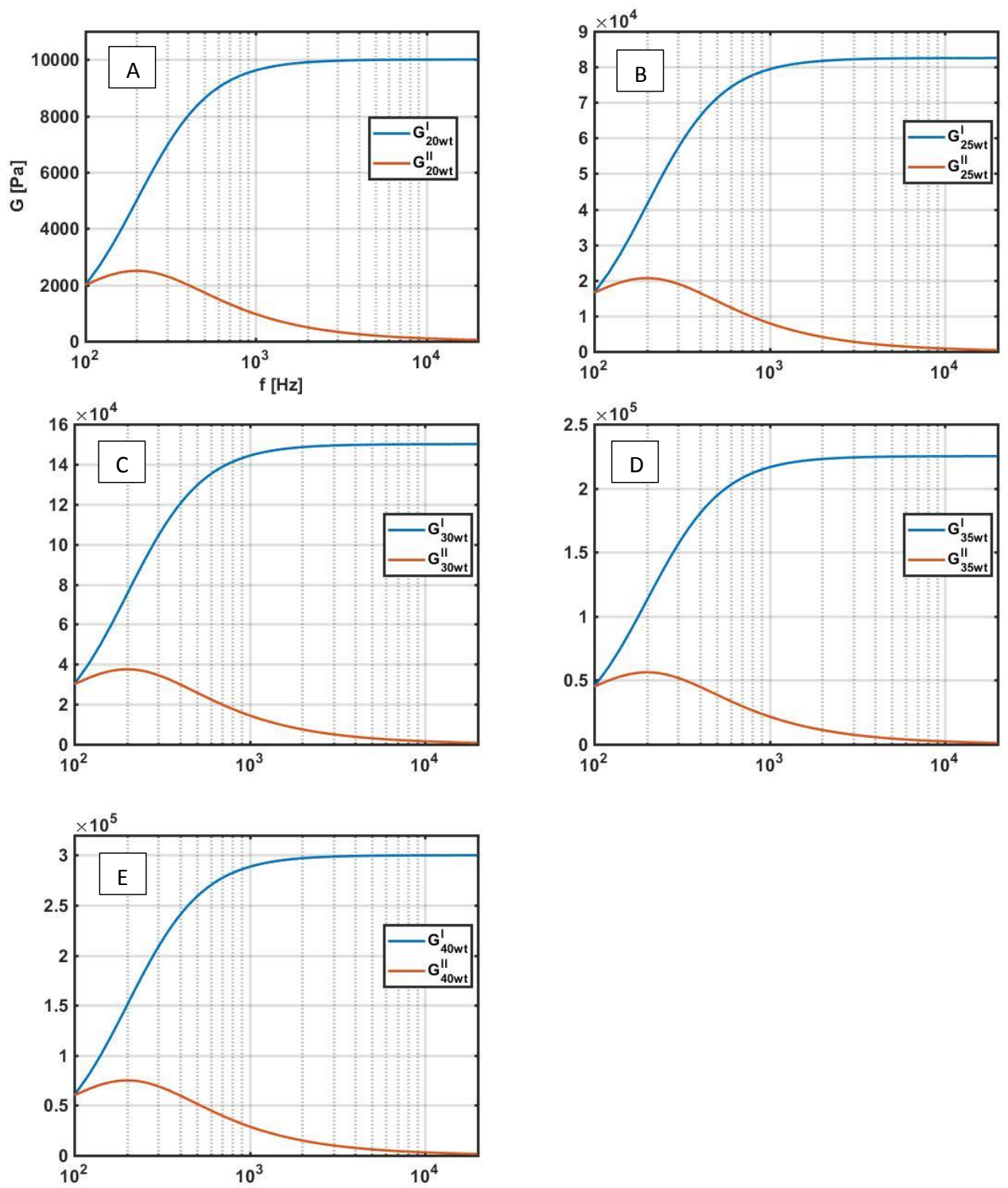

Fig. (6). Discrete relaxation spectrum. 
In order to reduce the computational cost of the numerical evaluation of the sound transmission loss, we focused on the main relaxation times of the discrete spectrum, as a higher number of relaxation times is typically negligible. The optimal number of relaxation times varies with the shear moduli of the viscoelastic system.

Regardless of the filler concentration, all the STFs share two main relaxation times $\left(10^{-2} \mathrm{~S}\right.$ and $\left.5 \mathrm{~S}\right)$, that can be traced back to the PEG200 carrier of the fluid.

The obtained spectra are compatible with the ones obtained by UV-visible assessment of hydrocluster formation in previous works $[24,25]$. As expected, all the fluids show the same relaxation times, as they share the same carrier, although the associated peaks with each relaxation time vary greatly for each fluid with the filler concentration, as it is controlled by the variation of the fluid viscosity with both shear strain and angular frequency.

As it can be inferred from the previous graphs, the error in the extrapolation of the relaxation times is higher for lower relaxation times (i.e. higher frequencies) and for higher filler concentration, as we deviate more and more from linear-viscoelasticity.

Finally, the extrapolated values for the shear moduli at higher regular frequency can be obtained by inserting the spectral data Fig. (7) into equation 3. In order to provide smoother input data for the following model, the results were filtered in terms of $1 / 3$ octave bands.

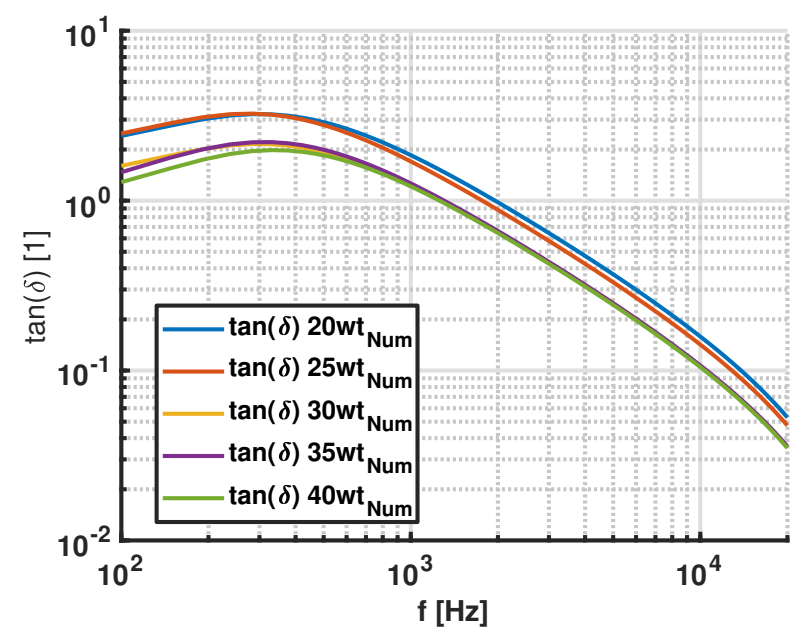

Fig. (7). High regular frequency extrapolation of the low regular frequency rheological data (a-e).

It is important to highlight that the low regular frequency damping loss factor does not alter significantly the high regular frequency response of the fluid for the application of interest, as this parameter affects only the skin-core-skin resonance of the panel.

The apparent viscosity allows to estimate qualitatively the variation of the relaxation strength for our fluids. Although the relaxation strength of the fluids doesn't change significantly with the filler concentration for samples with a wt higher than 30 , attesting approximately at 3.25 for the samples with a wt of 30,35 and 40. On the other hand, samples with a wt of 20 and 25 show a significantly lower relaxation strength, of 2.75. Therefore, we can expect that, samples with a 30 (or higher) wt will grant the CLD sandwich with a superior sound insulation, on an average.

However, the response associated to resonance phenomena must be analyzed from a different perspective.

More in detail, fluids with higher filler concentration exhibit both higher storage and loss moduli, as their apparent viscosity increases with angular frequency and strain and the Kramers-Krönig's integral equations link both these quantities. As a consequence, the filler concentration will alter the critical regular frequency of the panel, as stiffer panels tend to exhibit higher critical frequencies [26], with little regards to the loss modulus.

On the other hand, the actual transmission loss notches $(\triangle T L)$, are highly dependent on the damping loss factor Fig. (8) and, therefore, for fluids with lower wt, we can expect slightly higher $\tan (\delta)$, thus reducing the $\Delta T L$. 


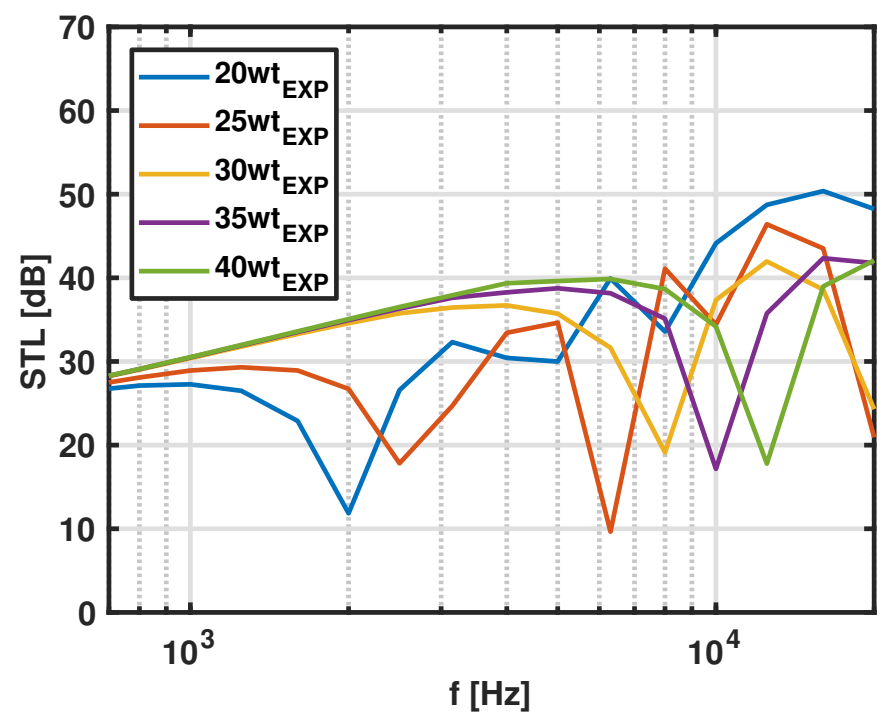

Fig. (8). High regular frequency extrapolation of the damping loss factor.

At last, as a confirmation of the significance of this extrapolation, it can be seen that the peak of the damping loss factor Fig. (8), influenced by the $T_{g}$ of the carrier fluid [27], as expected doesn't change significantly with increasing filler concentration.

\subsection{Insertion Loss Results}

The results of the insertion loss test Fig. (9) highlight the validity of the extrapolated results.
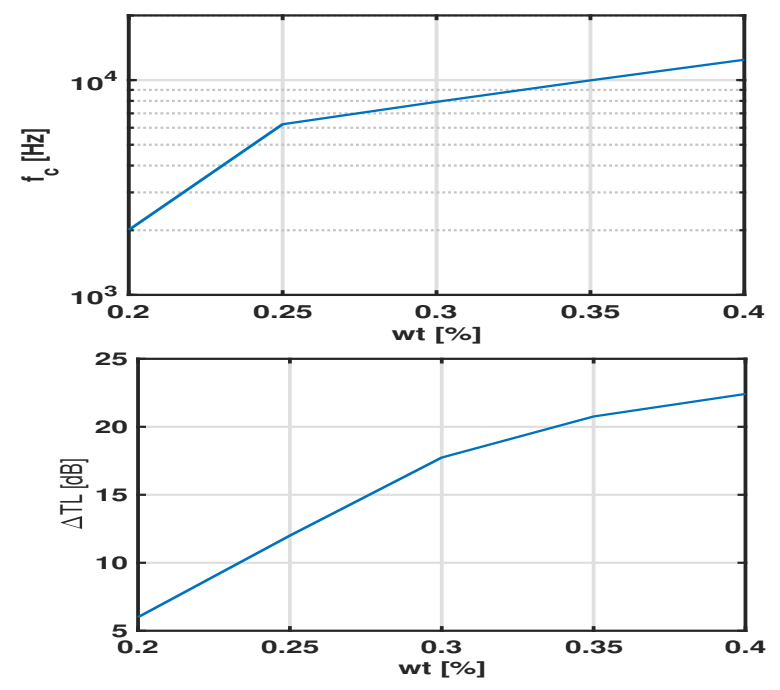

Fig. (9). Acoustic insertion loss test on stfs with different Wt.

As a matter of fact, samples with a high filler concentration (wt higher than 30), i.e. higher stiffness, tend to exhibit better performance below the coincidence regular frequency, because of a good compromise between a high viscosity, therefore of the higher relaxation strength. At the same time, they tend to exhibit steeper coincidence notches, as their damping loss factor at higher frequencies, in a range rounding the $\mathrm{f}_{\mathrm{c}}$, is lower than the other samples. At the same time, the notch associated to the critical regular frequency Fig. (10) does not vary remarkably for samples with a wt higher than 30, nearly reaching a plateau after this wt. On the other hand, the $f_{c}$ for a wt higher than 22 overcomes the upper bound of maximum sensitivity band of the human ear (i.e. $2000 \mathrm{~Hz}-4000 \mathrm{~Hz}$ ) [28] and therefore, even though the $S T L$ reduction is higher, this does not remarkably affect the end-user. Finally, for wt higher than 40, the coincidence regular frequency exits the audible regular frequency band. 


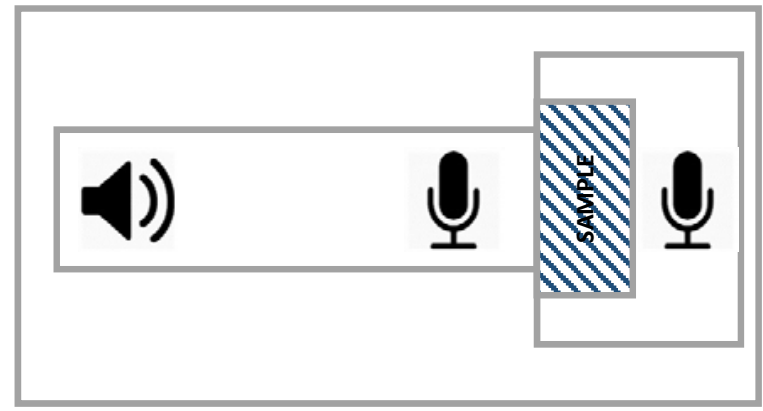

Fig. (10). Coincidence response $V s$ filler concentration.

\section{NUMERICAL MODELING OF THE CLD SANDWICH}

\subsection{GTMM Model}

The first approach used for the modeling of the phenomenon is one of the most commonly used in sound insulation analysis. The origins for this model stem from a simple $2 \mathrm{~d}$ analysis, in which a $3 \mathrm{~d}$, complex phenomenon acoustic is regarded as a classical optics problem Fig. (11). The main hypotheses beyond this model can be summarized in the following key points:

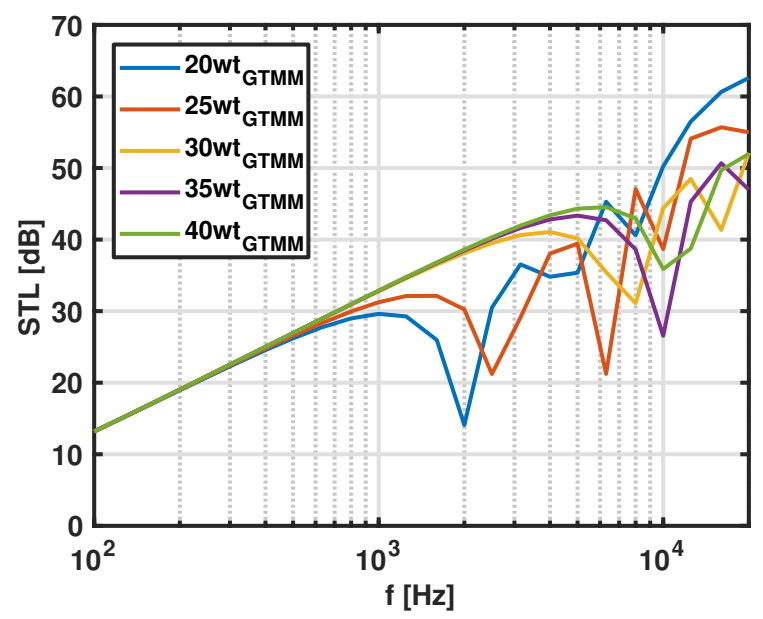

Fig. (11). Scheme of the gtmm model.

1. The influence of the door and interior attachments inside the measurement environment are negligible.

2. The specimen is placed in a perfectly rigid baffle.

3. The effect of the boundary conditions is negligible to the panel response (i.e. infinitely extended panel).

4. The real sound source is simplified as a planar sound field.

5. The core is made of a linear viscoelastic, isotropic and homogeneous material.

6. The skins are made of a linear elastic, isotropic homogeneous material.

To solve the problem, we used a Matlab script to evaluate the following integral identity:

\subsection{Equation 4- Definition of the Sound Transmission Loss of a Composite Structure}

Where:

- $\theta$ denotes the incidence angle of the pressure wave;

- $K$ denotes the wave number of the pressure wave;

- $Z$ denotes the acoustical impedence;

- $\omega$ denotes the acoustical transmissibility; 
The integration angle varies from 0deg to 78deg: this particular value derives form numerical considerations, as the contribution of angles higher than this are negligible to the sound insulation properties of the panel [29].

The results shown in Fig. (12) will be analyzed thoroughly in the following. We would like however to highlight some basic considerations. The GTMM correctly portrays the effect of the wt to the STL in the $1 \mathrm{kHz}$ to $10 \mathrm{KHz}$ band: the higher the wt, the higher the $S T L$ on an average. Furthermore, this model correctly described the effect of the wt to the $f_{c}$, as the coincidence notch gets closer to the maximum sensitivity range $(1000-4000 \mathrm{~Hz})$ as we decrease the wt Finally, this oversimplified model correctly suggests, that as we increase the wt, the lower the STL gets after the coincidence.

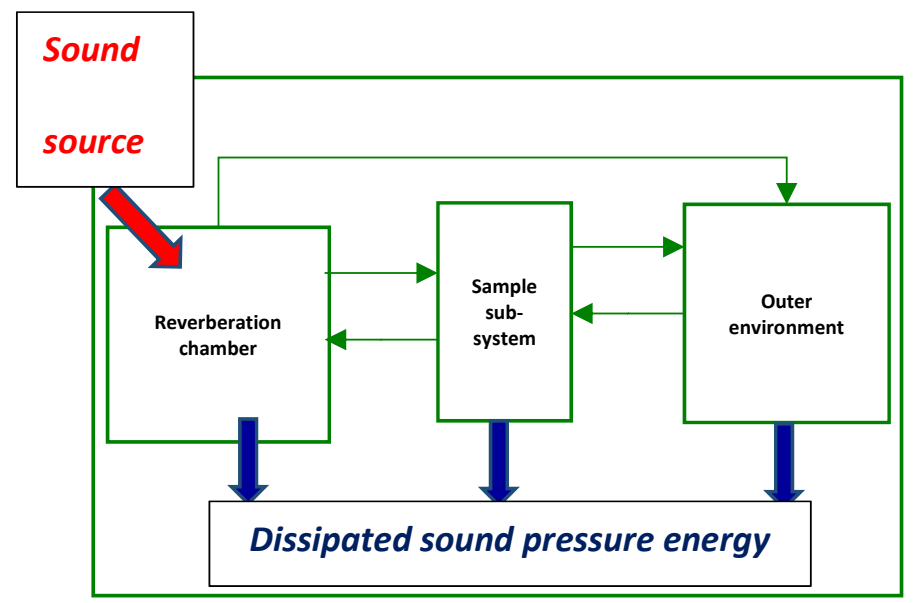

Fig. (12). GTMM model results for the $S T L$

However, there is one major incongruence deriving from the fact that it does not correctly model the actual notch nearby the $f_{c}$. This model in fact would suggest the notch decreases with the wt, while insertion loss tests suggest otherwise (Fig. 10).

Even though this approach is computationally inexpensive, regardless of the CLD sandwich dimension and material properties, it is inherently limited by the fact that it neglects the effect deriving from both the sound source and the boundary conditions, as it approximates the sample as an infinitely extended planar surface. This error becomes more and more remarkable at higher frequencies or, in other terms, when the wavelength becomes compatible to the sample thickness.

\subsection{SEA Model}

In order to compensate the error introduced by the diffused sound field condition and the neglected boundary conditions, we choose to model our system by means of a technique capable of carrying out a high regular frequency analysis and discretizing the geometrical testing environment. FEM models typically can't be used to model high regular frequency Vibroacoustic phenomena, as the low wavelength involved usually require an higher number of seeds for the correct discretization (ideally a number higher than [30]). As the high frequencies involved in this study involve a great number of modal contributions, we used a SEA approach.

Contrarily to the previous model, the hypotheses involved in this study can be summarized in the following points:

- The reverberation chamber is treated as a cavity with perfectly reflecting walls.

- The porous absorption of the walls of the reverberation chamber was neglected, as the micro porosities of the wall prove to be relevant to the STL evaluation at frequencies higher than $20 \mathrm{kHz}$ [31].

- Attachments inside the reverberation chamber are neglected (i.e. we neglect the fixture that hold the microphones and the speakers).

- The specimen is placed in a perfectly rigid baffle.

- The real sound source is treated as a monopole ball pulse source.

This approach however correctly takes into account the finite dimension of the testing environment and the sample, thus providing better results at higher frequencies, incompatible with finite difference and finite element methods. In 
order to solve the equations associated to this study, we used for our model the open source Matlab library SEAlab [32].

We divided our phenomenon in a system made of three different subsystems (Fig. 13), i.e. the sample, the reverberation chamber and the outer environment) and we studied the statistical contribution of the various natural modes of the system to the energy flux between the three subsystems.

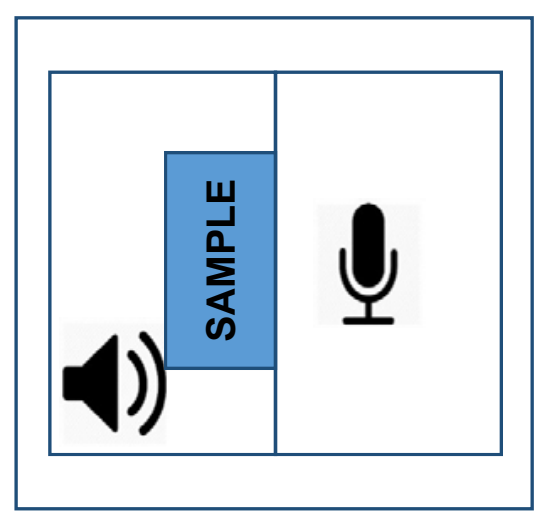

Fig. (13). GTMM model results for the $S T L$

The results obtained from the SEA model correctly portray the effect of the wt to the average $S T L$, as a higher wt grant higher sound insulation properties.

Yet again, it correctly portrays the effect of the wt to the $f_{c}$. In fact, the lower the wt, the closer is the coincidence notch to the band 1000-4000 Hz. Furthermore, the SEA model correctly predicts that samples with lower wt perform better after the coincidence.

Finally, this model is congruent with the experimental results at higher frequencies, as the notch actually increases and moves to the right with the wt (Fig. 14).

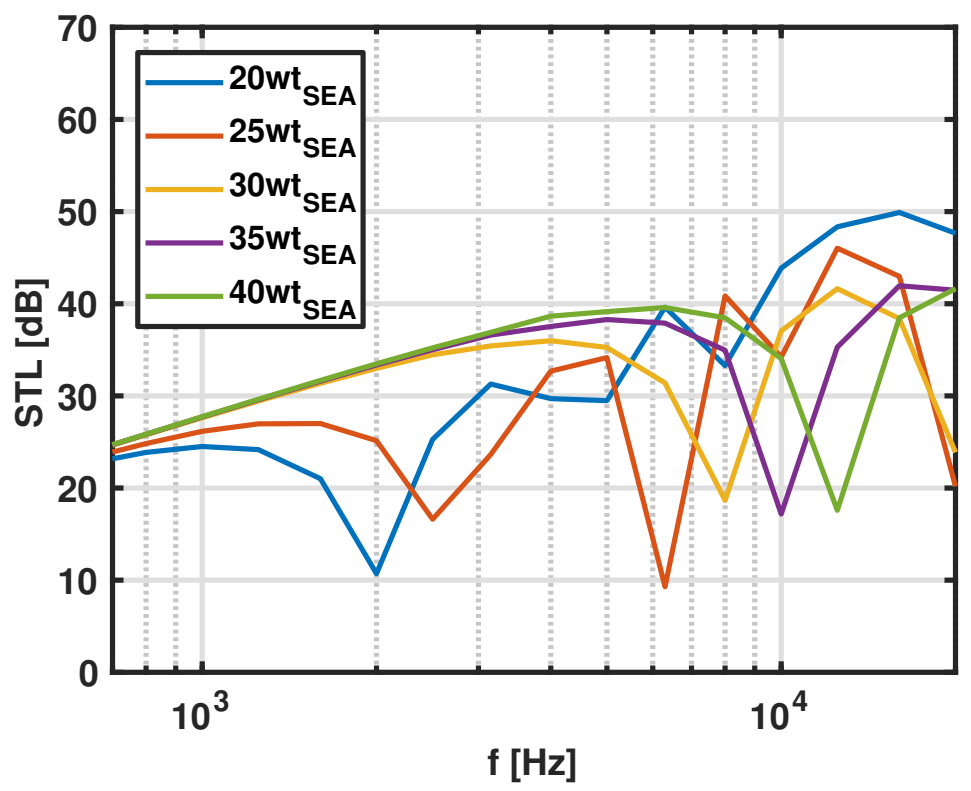

Fig. (14). Sea model results for the $S T L$

\section{RESULTS COMPARISON}

These results are summarized in the following comparison plots Fig. (15), where we super-impress the results obtained for the GTMM model, the SEA model and the IL experimental data. 
A

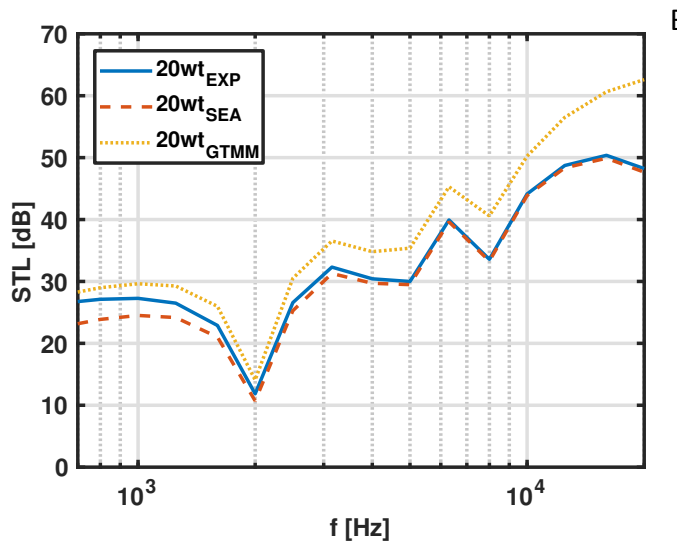

C

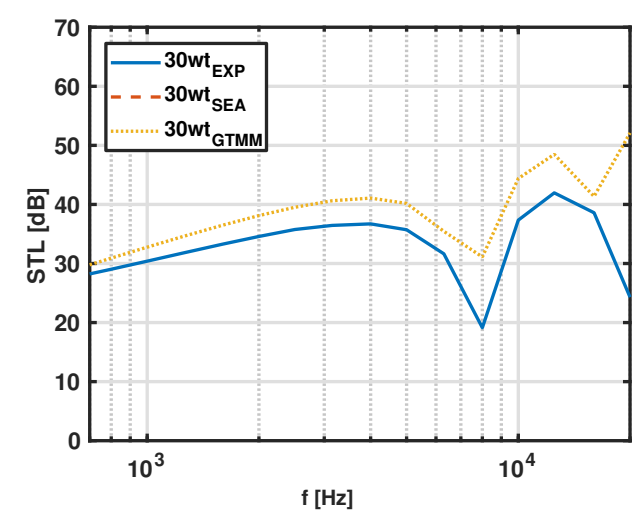

E

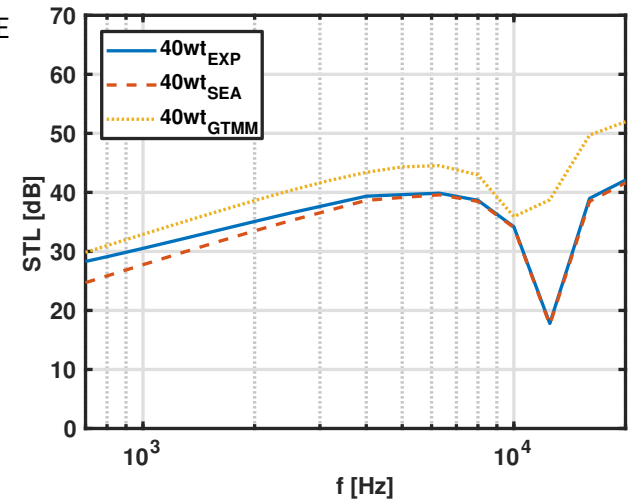

B

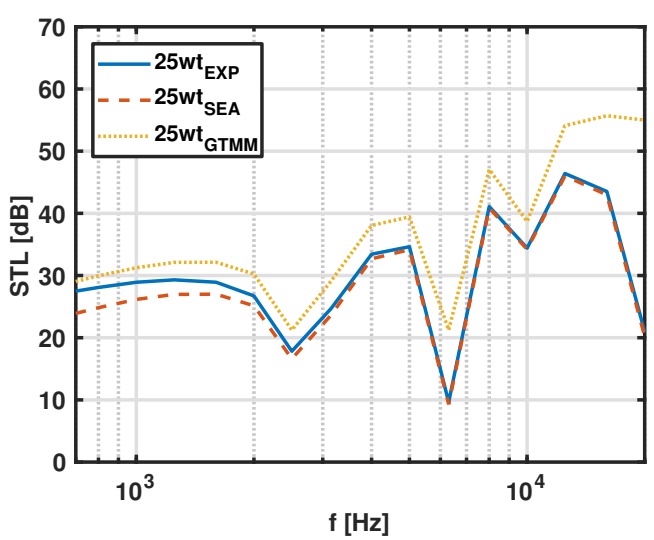

D

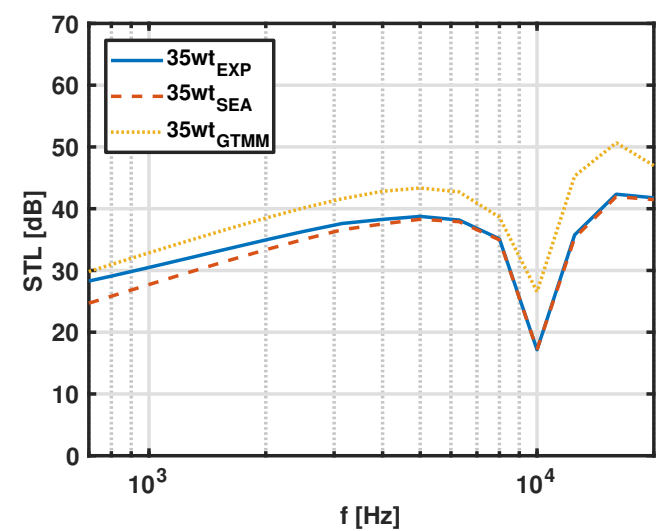

Fig. (15). Results comparison for the various samples (A-E)

The IL experimental results are in a good agreement with the new SEA model, even though there is a discrepancy at higher frequencies. The discrepancy is lower before $f_{c}$ for the GTMM model and above the $f_{c}$ for the SEA model. The difference at higher frequencies for both the models can be ascribed, first of all, to the numerical extrapolation of the shear moduli, that becomes more and more unreliable at higher frequencies, especially with fluids with a higher wt (i.e. wider relaxation band and higher non-linearities). Secondarily, SEA models, and statistical approaches as a whole, need a sufficiently large population to provide accurate results and, therefore, need a higher number of modal contributions (i.e. higher frequencies).

However, the discrepancy between SEA and IL data is always lower than $6 \%$.

\section{CONCLUSION}

The objective of this work was the study of the sound insulatio characterized by a viscosity that increases with the in taneous shear rate.
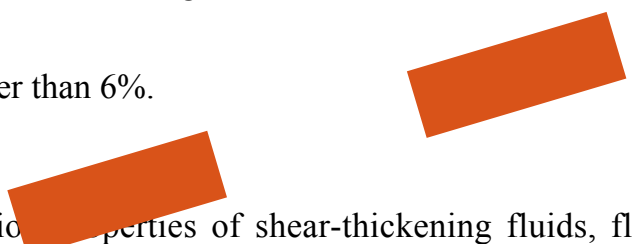
These Non-Newtonian fluids were used for the manufacturing of sandwich panels in constrained layer damping configuration.

The first part of this work was focused on the analysis of the linear viscoelastic region for these materials, by means of a 2-step rheological analysis. Successively, the data obtained was used for the extrapolation of the continuous relaxation spectrum and to calculate the discrete relaxation spectrum (i.e. relaxation times and their associated peak values) and, therefore, to model the acoustic constitutive behavior of these materials.

Secondarily, the sound insulation properties of the sandwich panels were measured by means of sound insertion loss measurements.

Finally, modeled the sound insulation properties of our materials by means of a SEA numerical approach and we compared the obtained results with both the experimental results and with the GTMM, a well-established numerical approach for Vibroacoustic phenomena in the current literature.

\section{CONSENT FOR PUBLICATION}

Not applicable.

\section{CONFLICT OF INTEREST}

The authors declare no conflict of interest, financial or otherwise.

\section{ACKNOWLEDGEMENTS}

Declared none.

\section{REFERENCES}

[1] Ding J, Tracey P, Li W, Peng G, Whitten PG, Wallace GG. Review on shear thickening fluids and applications 2013; XX: 1-12.

[2] Liao F-S, Hsu T-CJ. Prediction of vibration damping properties of polymer-laminated steel sheet using time-temperature superposition principle. J Appl Polym Sci 1992; 45: 893-900.

[http://dx.doi.org/10.1002/app.1992.070450516]

[3] Fischer C, Bennani A, Michaud V, Jacquelin E, Månson J-AE. Structural damping of model sandwich structures using tailored shear thickening fluid compositions. Smart Mater Struct 2010; 19: 35017.

[http://dx.doi.org/10.1088/0964-1726/19/3/035017]

[4] Fischer C, Plummer CJG, Michaud V, Bourban PE, Månson JAE. Pre- and post-transition behavior of shear-thickening fluids in oscillating shear. Rheol Acta 2007; 46: 1099-108. [http://dx.doi.org/10.1007/s00397-007-0202-y]

[5] Mahjoob MJ, Mohammadi N, Malakooti S. An investigation into the acoustic insulation of triple-layered panels containing Newtonian fluids: Theory and experiment. Appl Acoust 2009; 70: 165-71.

[http://dx.doi.org/10.1016/j.apacoust.2007.12.002]

[6] Waters KR, Mobley J, Miller JG. Causality-imposed (Kramers-Kronig) relationships between attenuation and dispersion. IEEE Trans Ultrason Ferroelectr Freq Control 2005; 52(5): 822-33. [http://dx.doi.org/10.1109/TUFFC.2005.1503968] [PMID: 16048183]

[7] Raghavan SR. Shear-induced microstructural changes in flocculated suspensions of fumed silica. J Rheol (NYNY) 1995; 39 : 1311. [http://dx.doi.org/10.1122/1.550638]

[8] Chryss AG, Bhattacharya SN, Pullum L. Rheology of shear thickening suspensions and the effects of wall slip in torsional flow. Rheol Acta 2005; 45: 124-31. [http://dx.doi.org/10.1007/s00397-005-0001-2]

[9] Weese J. A reliable and fast method for the solution of Fredhol integral equations of the first kind based on Tikhonov regularization. Comput Phys Commun 1992; 69: 99-111.

[http://dx.doi.org/10.1016/0010-4655(92)90132-I]

[10] Cecchini F, Cherubini V, Nanni F. A D.O.E. analysis of the rheological properties of non-Newtonian fluids with different compositions 2016.

[11] Cecchini F, Fabbrocino F, Nanni F. Vibroacoustic response of a sandwich panel with a shear-thickening core.Compos Eng ICCE-25. Rome 2017 ; p. 32.

[12] Cecchini F, Cherubini V, Sadaf M, Fabbrocino F, Nanni F. Design of a puncture-resistant composite shell comprising a non-Newtonian core. Polym Test 2018. [http://dx.doi.org/10.1016/j.polymertesting.2018.03.019]

[13] Hasanzadeh M, Mottaghitalab V, Rezaei M. Rheological and viscoelastic behavior of concentrated colloidal suspensions of silica 
nanoparticles: A response surface methodology approach. Adv Powder Technol 2015.

[http://dx.doi.org/10.1016/j.apt.2015.08.011]

[14] Fischer C, Braun S A, Bourban PE, Michaud V, Plummer CJG, Månson JE. Dynamic properties of sandwich structures with integrated shearthickening fluids. Smart Mater Struct 2006; 15: 1467-75. [http://dx.doi.org/10.1088/0964-1726/15/5/036]

[15] Raghavan SR, Khan SA. Shear-Thickening response of fumed silica suspensions under steady and oscillatory shear. J Colloid Interface Sci 1997; 185(1): 57-67. [http://dx.doi.org/10.1006/jcis.1996.4581] [PMID: 9056301]

[16] Negi AS, Osuji CO. New insights on fumed colloidal rheology-shear thickening and vorticity-aligned structures in flocculating dispersions. Rheol Acta 2009; 48: 871-81.

[http://dx.doi.org/10.1007/s00397-008-0341-9]

[17] Fischer C. Shear thickening fluids as a tunable damping element: Experimental results and modeling - art. no. 652513 Act Passiv Smart Struct Integr Syst 2007; $6525: 52513$. Artn 652513 \n [http://dx.doi.org/Doi 10.1117/12.715833]

[18] Mahjoob MJ, Mohammadi N, Malakooti S. An investigation into the acoustic insulation of triple-layered panels containing Newtonian fluids: Theory and experiment. Appl Acoust 2009; 70: 165-71. [http://dx.doi.org/10.1016/j.apacoust.2007.12.002]

[19] Honerkamp J, Weese J. A nonlinear regularization method for the calculation of relaxation spectra. Rheol Acta 1993; 32 : 65-73. [http://dx.doi.org/10.1007/BF00396678]

[20] Weese J. A regularization method for nonlinear ill-posed problems. Comput Phys Commun 1993; 77: 429-40. [http://dx.doi.org/10.1016/0010-4655(93)90187-H]

[21] Elster C, Honerkamp J, Weese J. Using regularization methods for the determination of relaxation and retardation spectra of polymeric liquids. Rheol Acta 1992; 31: 161-74. [http://dx.doi.org/10.1007/BF00373238]

[22] Kontogiorgos V. Calculation of relaxation spectra from mechanical spectra in MATLAB. Polym Test 2010; 29: 1021-5. [http://dx.doi.org/10.1016/j.polymertesting.2010.09.007]

[23] Kontogiorgos V, Jiang B, Kasapis S. Numerical computation of relaxation spectra from mechanical measurements in biopolymers. Food Res Int 2009; 42: 130-6.

[http://dx.doi.org/10.1016/j.foodres.2008.09.005]

[24] Fahool M, Sabet AR. Relaxation time assessment in nanoparticle containing bimodal and mono-disperse shear thickening fluids Sci Iran Trans F, Nanotechnol 2016; 23: 1496.

[25] Fahool M, Sabet AR. UV-visible assessment of hydrocluster formation and rheological behaviour in bimodal and mono-disperse shear thickening fluids. Rheol Acta 2015; 54: 77-83. [http://dx.doi.org/10.1007/s00397-014-0821-z]

[26] Narang PP. A theoretical study of sound transmission through aerogel glazing systems. Appl Acoust 1991; $34: 249-59$. [http://dx.doi.org/10.1016/0003-682X(91)90008-3]

[27] Corsaro RD, Sperling LH. Sound and vibration damping with polymers. In: Sound Vib. Damping with Polym 1990; pp. 5-22.http://agris.fao.org/agris-search/search.do?recordID=US201300263178

[28] Rossing TD. Springer Handbook of Acoustics, Book. 2007; p. 1182. [http://dx.doi.org/10.1007/978-0-387-30425-0.]

[29] Assaf S, Guerich M. Numerical prediction of noise transmission loss through viscoelastically damped sandwich plates. J Sandw Struct Mater 2008; 10: 359-84

[http://dx.doi.org/10.1177/1099636207088444]

[30] Loredo A, Plessy A, El Hafidi A, Hamzaoui N. Numerical vibroacoustic analysis of plates with constrained-layer damping patches. J Acoust Soc Am 2011; 129(4): 1905-18. [http://dx.doi.org/10.1121/1.3546096] [PMID: 21476647]

[31] Del Rey R, Alba J, Bertó L, Gregori A, Gregori A. Small-sized reverberation chamber for the measurement of sound absorption. Mater Constr 2017; 67: 139.

[http://dx.doi.org/10.3989/mc.2017.07316]

[32] Johansson D. Statistical Energy Analysis software, Technology. 2010.http://www.ta.chalmers.se/downloads/open/research/ sealab/Master Thesis - Daniel Johansson Peter Comnell.pdf Accessed March 15, 2017

(C) 2018 vibroacoustics et al.

This is an open access article distributed under the terms of the Creative Commons Attribution 4.0 International Public License (CC-BY 4.0), a copy of which is available at: (https:/creativecommons.org/licenses/by/4.0/legalcode). This license permits unrestricted use, distribution, and reproduction in any medium, provided the original author and source are credited. 